\title{
HYDROSTATIC-PRESSURE DEEP LEVEL TRANSIENT SPECTROSCOPY STUDY OF THE HETEROANTISITE ANTIMONY LEVEL IN GaAs*
}

\author{
A. Babiński, J. Przybytek, M. BaJ \\ Institute of Experimental Physics, Warsaw University \\ Hoża 69, 00-681 Warszawa, Poland \\ P. Omling, L. Samuelson
}

Department of Solid State Physics, University of Lund, Box 118, 22100 Lund, Sweden

AND T. SLUPIŃSKI

Laboratory of Crystal Growth Physics, Przyokopowa 28, 01-208 Warszawa, Poland

We present some preliminary results of the first hydrostatic-pressure study of the electronic level related to the Sb-heteroantisite defect in GaAs. We studied two kinds of $n$-type GaAs samples doped with antimony: bulk samples grown by liquid encapsulated Czochralski method and thin layers grown by metalorganic chemical vapour deposition technique. We found strongly nonlinear pressure dependence of the activation energy of the emission rate for the level. Moreover, the results obtained for the bulk material were faiily different from those obtained for thin metalorganic chemical vapour deposition layers. The possible explanation of this difference is presented.

PACS numbers: $71.55 . E q, 62.50 .+p$

Antisite-type defects are very important and interesting point defects in III-V semiconductors. The best known defect of such a type is EL2 in GaAs. Recently some works on antimony heteroantisite defect in GaAs have been published [1-6]. It has been shown that such a defect has a donor level about $0.5 \mathrm{eV}$ below the conduction band edge of GaAs. We performed the first measurements of the hydrostatic pressure dependence of the $\mathrm{Sb}_{\mathrm{Ga}}(0 /+)$ level activation energy in order to compare it with the pressure coefficient of EL2.

*This work was supported in part by the Committee for Scientific Research grant 201799101. 
Two types of samples were investigated: bulk material ( $n$-type, Te, Sb doped, $n=2 \div 8 \times 10^{16} \mathrm{~cm}^{-3}$ ), which was grown by means of LEC method at the Laboratory of Crystal Growth Physics, Warsaw, and thin layers (Sb doped, $n$-type, $n=3 \div 4 \times 10^{14} \mathrm{~cm}^{-3}$ ), which were grown by MOCVD technique at the Department of Solid State Physics, University of Lund. Two bulk samples were measured: B11C5 - from the beginning and B11S5 - from the tail of a crystal. The DLTS measurements were performed by means of isothermal frequency scan mode [7]. The measurements performed under high pressure up to $1.0 \mathrm{GPa}$ were done in high-pressure cell with benzene as a pressure transmitting medium.
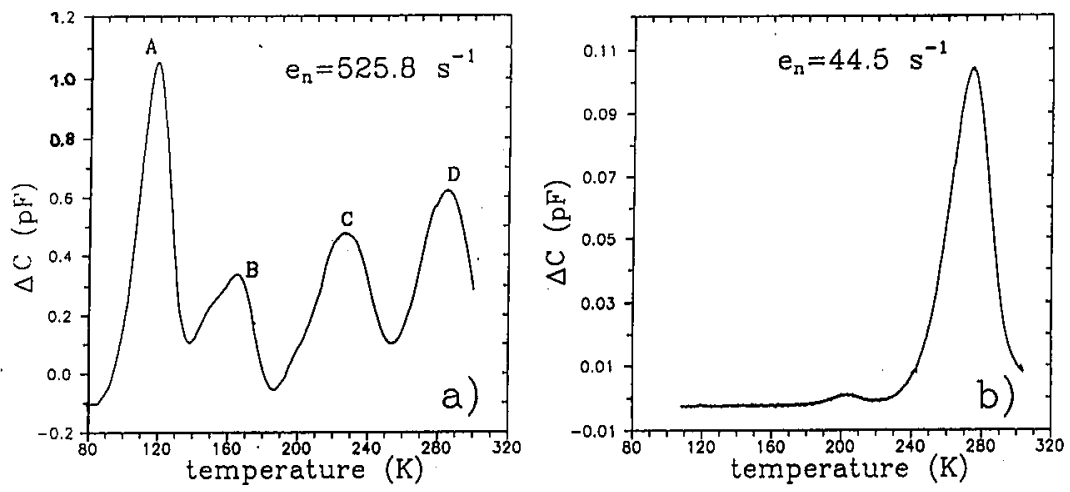

Fig. 1. (a) DLTS spectrum of a LEC GaAs:Sb,Te sample below $300 \mathrm{~K}$. (b) DLTS spectrum of a MOCVD GaAs:Sb sample below $300 \mathrm{~K}$.

There were 5 DLTS peaks detected in a bulk material (peaks below $300 \mathrm{~K}$ are presented in Fig. 1a). The emission-rate activation energies of investigated levels were as follows: $A-\Delta E=0.22 \mathrm{eV}, B-\Delta E=0.28 \mathrm{eV} ; C-\Delta E=0.52 \mathrm{eV}$; $D-\Delta E=0.50-0.52 \mathrm{eV} ; E(E L 2)-\Delta E=0.81 \mathrm{eV}$. Although the activation energies of both $C$ and $D$ levels were close to the value reported for $\mathrm{Sb}_{\mathrm{Ga}}(0 /+)$ level [2], however, the capture cross-section of the $C$ level was enormously large $\left(\sigma_{0}=6 \times 10^{-11} \mathrm{~cm}^{2}\right)$ and likely corresponded to a more complicated defect than the $\mathrm{Sb}_{\mathrm{Ga}}$ one. Therefore we concluded that this was peak $D$ which originated from the $\mathrm{Sb}_{\mathrm{Ga}}(0 /+)$ level. The concentration of $D$ level was $0.4 \div 1.2 \times 10^{15} \mathrm{~cm}^{-3}$. The results of DLTS measurements performed under high pressure are presented in Fig. 2a. The dependence of the activation energy of the $D$ level emission rate was strongly nonlinear and depended on a sample. The best fits to a quadratic polynomial were - for B11S sample:

$$
\begin{aligned}
& \Delta E(p)=\Delta E(0)-162[\mathrm{meV} / \mathrm{GPa}] \times P+52\left[\mathrm{meV} / \mathrm{GPa}^{2}\right] \times P^{2} \text { at } 295.4 \mathrm{~K}, \\
& \Delta E(p)=\Delta E(0)-158[\mathrm{meV} / \mathrm{GPa}] \times P+50\left[\mathrm{meV} / \mathrm{GPa}^{2}\right] \times P^{2} \text { at } 302.9 \mathrm{~K},
\end{aligned}
$$
and for B11C sample:

$$
\Delta E(p)=\Delta E(0)-150[\mathrm{meV} / \mathrm{GPa}] \times P+70\left[\mathrm{meV} / \mathrm{GPa}^{2}\right] \times P^{2} \text { at } 294.7 \mathrm{~K} .
$$

For comparison the pressure dependence of the activation energy of the $C$ level emission rate (perfectly linear!) is also presented in Fig. 2a. The pressure coefficient for this level measured at $236.1 \mathrm{~K}$ was equal to $111 \mathrm{meV} / \mathrm{GPa}$. 

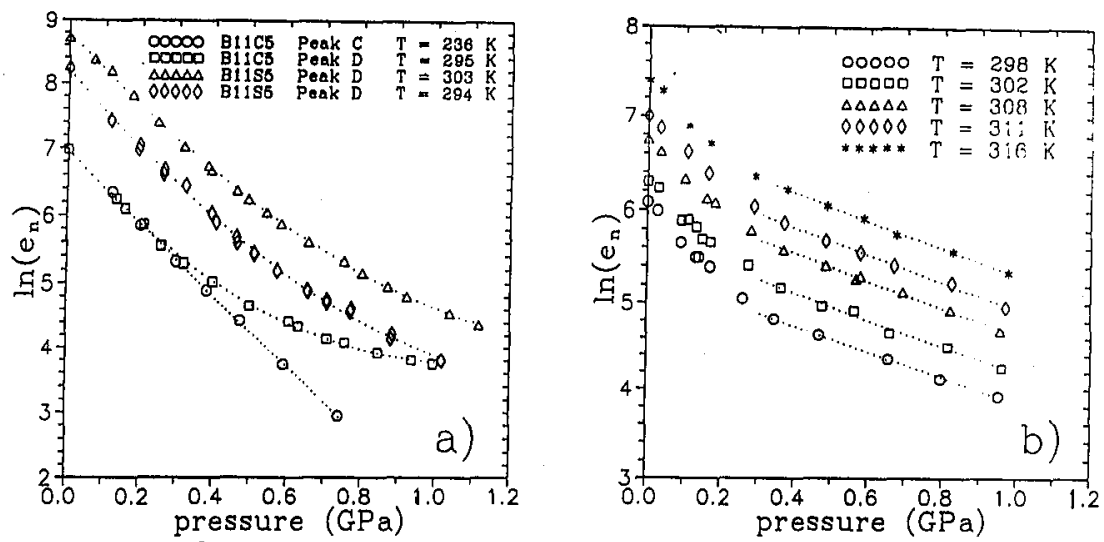

Fig. 2. (a) The pressure dependence of electron emission rate of the $D$ and $C$ levels measured in LEC GaAs:Sb,Te samples. (b) The pressure dependence of electron emission rate of the $\mathrm{Sb}_{\mathrm{Ga}}(0 /+)$ level measured in MOCVD GaAs:Sb sample.

The identification of $\mathrm{Sb}_{\mathrm{Ga}}(0 /+)$ level in MOCVD layers was much easier than in bulk samples, because there were only lwo DLTS peaks detected in MOCVD samples (and one of them was related to EL2!) - see Fig. 1b. The signature of the $\mathrm{Sb}_{\mathrm{Ga}}(0 /+)$ level was $\Delta E=0.58 \mathrm{eV}$ and $\sigma_{0}=1.7 \times 10^{-13} \mathrm{~cm}^{2}$. The concentration of this level was equal to $4 \times 10^{13} \mathrm{~cm}^{-3}$. The results of DLTS measurements performed under pressure are presented in Fig. 2b. As it can be seen, for MOCVD sample the dependence of the activation energy of the emission rate is also nonlinear. However, for pressure higher than $0.3 \mathrm{GPa}$ straight lines fit the experimental data quite well. For all the temperatures the slopes of these lines were found to be equal to $40 \pm 3 \mathrm{meV} / \mathrm{GPa}$. This value appears to be in good agreement with the data reported for EL2 [8, 9].

Some intriguing facts follow from our experimental data. There are mainly: 1) pressure dependence of the activation energy of $\mathrm{Sb}_{\mathrm{Ga}}(0 /+)$ level emission rate is strongly nonlinear; 2) while the values of the emission rates of the $\mathrm{Sb}_{\mathrm{Ga}}(0 /+)$ level in bulk samples are of the same order as these of thin layers, the pressure dependence of the emission rates for both kinds of samples is distinctly different; 3 ) high pressure parts $(P>0.7 \mathrm{GPa})$ of the data presented in Fig. $2 \mathrm{a}$ and $2 \mathrm{~b}$ seem to converge, therefore both of the absolute values of the emission rates and their pressure dependences become very similar for both kinds of samples.

The above facts are not understood as yet, unfortunately. However, it should be pointed out that in order to obtain information about the $\mathrm{Sb}_{\mathrm{Ga}}$ defect itself one has to separate the sample dependent properties.

We propose that those sample dependent properties might be due to the influence of the electric field in Schottky barrier depletion layers. For example we found that the $\mathrm{Sb}_{\mathrm{Ga}}$ related peak occurred at different temperatures depending on bias and saturation voltage. Moreover, the electric field in depletion layers of MOCVD samples was much lower (much lower free-electron concentration) than 
for bulk samples. This might explain the difference in the experimental results for both kinds of samples and suggests that the results for MOCVD samples should be treated as more reliable.

The pressure dependence of the activation energy of the $\mathrm{Sb}_{\mathrm{Ga}}(0 /+)$ level, obtained for MOCVD samples at higher pressures is similar to that of EL2 level $[8,9]$. The dramatical change of the pressure coefficient near $0.3 \mathrm{GPa}$ may be due to the capture cross-section activation-energy dependence. However, the direct measurements of this activation energy were unsuccessful.

\section{References}

[1] W.C. Mitchel, P.W. Yu, J. Appl. Phys. 57, 623 (1985).

[2] W.C. Mitchel, P.W. Yu, J. Appl. Phys. 62, 4781 (1987).

[3] M. Bäumler, F. Fuchs, U. Kaufman, Phys. Rev. B 40, 8072 (1989).

[4] M. Bäumler, J. Schneider, U. Kaufmann, W.C. Mitchel, P.W. Yu, Phys. Rev. B 39, 6253 (1989).

[5] M.J. Caldas, J. Dąbrowski, A. Fazzio, M. Scheffler, Phys. Rev. Lett. 65, 2046 (1990).

[6] R. Yakimova, P. Omling, B.H. Yang, L. Samuelson, J.-O. Fornell, L. Ledebo, Appl. Phys. Lett. 59, 1323 (1990).

[7] G. Ferenczi, J. Boda, T. Pavelka, Phys. Status Solidi A 94, K119 (1986).

[8] L. Dobaczewski, A. Sienkiewicz, Acta Phys. Pol. A71, 341 (1987).

[9] A. Zylbersztejn, R.H. Wallis, J.M. Besson, Appl. Phys. Lett. 32, 764 (1978). 